Article

\title{
Computational Fluid Dynamic Analysis of a Floating Offshore Wind Turbine Experiencing Platform Pitching Motion
}

\author{
Thanhtoan Tran ${ }^{1}$, Donghyun Kim ${ }^{1} *$ and Jinseop Song ${ }^{2}$
}

1 Graduate School of Mechanical and Aerospace Engineering, Gyeongsang National University (GNU), 900 Gazwa, Jinju 660-701, Gyeongnam, Korea; E-Mail: trantoan2008@ gnu.ac.kr

2 Department of System Engineering, Korea Institute of Machinery and Materials (KIMM), Taejon 305-343, Korea; E-Mail: jssong@kimm.re.kr

* Author to whom correspondence should be addressed; E-Mail: dhk@gnu.ac.kr; Tel.: +82-55-755-2083; Fax: +82-55-755-2081.

Received: 14 May 2014; in revised form: 25 July 2014 / Accepted: 29 July 2014 /

Published: 4 August 2014

\begin{abstract}
The objective of this study is to illustrate the unsteady aerodynamic effects of a floating offshore wind turbine experiencing the prescribed pitching motion of a supporting floating platform as a sine function. The three-dimensional, unsteady Reynolds Averaged Navier-Stokes equations with the shear-stress transport (SST) k- $\omega$ turbulence model were applied. Moreover, an overset grid approach was used to model the rigid body motion of a wind turbine blade. The current simulation results are compared to various approaches from previous studies. The unsteady aerodynamic loads of the blade were demonstrated to change drastically with respect to the frequency and amplitude of platform motion.
\end{abstract}

Keywords: floating offshore wind turbine; Reynolds Averaged Navier-Stokes; dynamic mesh; overset grid approach; Fatigue, Aerodynamics, Structures, and Turbulence (FAST)

\section{Introduction}

The flow field around a wind turbine is often modeled as a steady, uniform, axial wind. In reality, the flow around a turbine blade is a more complex process that includes misalignments, wind shear, turbulence and dynamic winds. For a floating offshore horizontal axis wind turbine (HAWT), this process becomes more complex than for an onshore or fixed offshore system. Because of the motion of floating wind turbine, which includes three translational components (heavy in the vertical, sway in the 
lateral and surge in the axial) and rotational components (yaw about the vertical axis, pitch about the lateral, and roll about the axial) as shown in Figure 1, the additional effect of the contribution of a wind is accounted for in the turbine system. In those motions, platform pitching and yawing motion primarily lead to a non-uniform or skewed flow on a rotor turbine and may cause significant effects on the flow field around the rotor blade [1-4]. As an example, a typical floating HAWT (Figure 2, from left to right) shows a flow field around a rotor blade during spar-buoy pitching. As the rotor blade begins to pitch back, it interacts with its own wake which develops turbulence region. At several points, a toroidal recirculation is generated that flows normal to the rotor blade. This transitional aerodynamic phenomenon is called the vortex ring state, or settling with power [5]. The pitching motion intermediately causes a transient flow condition which is one of the potential operating and the simulation problems for a floating wind turbine. In particular, the pitching and yawing motion may cause more power in a floating offshore HAWT system because of the above issues $[4,6,7]$.

Figure 1. Degrees of freedom for an offshore floating wind turbine platform.

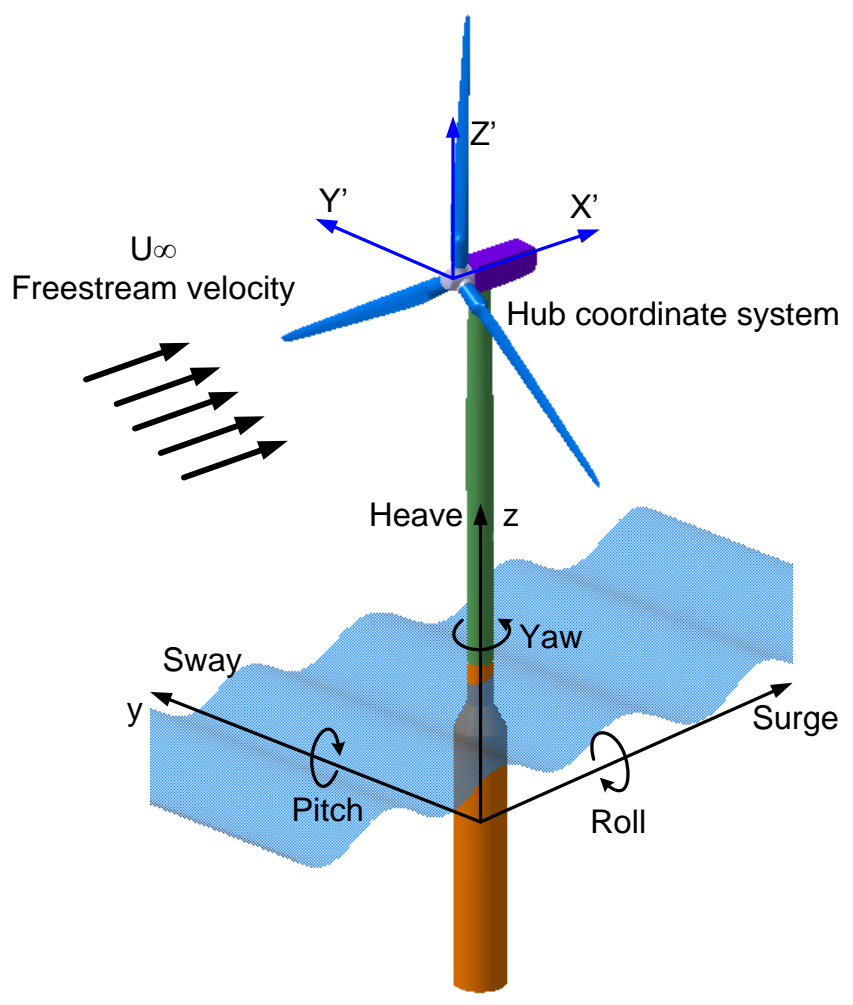

The major objective of the present work is to show the different predictions among traditional blade element momentum, general dynamic wake and advanced computational fluid dynamics approach for a floating offshore wind turbine (FOWT). In this study, Phase IV of the IEA Annex XXIII Offshore Code Comparison Collaboration (OC3) [8], which considered the spar-buoy concept, was chosen to show the critical effects of unsteady aerodynamic loads because of platform motion. The computational fluid dynamics (CFD) analysis of a three-dimensional unsteady flow analysis of a 5-MW floating offshore wind turbine was performed to consider the effects of the pitching degrees of freedom (DOF) motion of the support platform. Using computational fluid dynamics, the Reynolds Averaged Navier-Stokes equation with the shear-stress transport (SST) $k$ - $\omega$ turbulence model was applied. This model has the potential to provide consistent and physical simulations of the complex 
flow field around a wind turbine blade [9-11]. The meshes are used to discretize a computational domain that contains several different meshes that overlap each other in an arbitrary manner. Rigid Body Motion (RBM) was used to model the rotor blades in which the computational domain is changing with time because of motion on the blade surface boundaries. The superposing motions technique that allows you to add more rigid rotations to translation and rotation motions was applied herein. In the present study, blade rotation motion was added to the platform pitching motion that was defined as a sine function. Superposing motion can be simultaneously applied for one or more arbitrary motions. The current results are also compared to a developed in-house code based on the unsteady blade element momentum (UBEM) theory with the direct local relative velocity method (DLRM) that was constructed in a previous study [12]. We also proposed the equivalent averaged velocity method (EqAM) that can simplifies the relative velocity calculations on a rotating blade due to the platform pitching motion as the relative velocity at a hub instead of calculating the direct local velocity at each blade segment. Using EqAM, both in-house UBEM and FAST (Fatigue, Aerodynamics, Structures, and Turbulence) code with AeroDyn module of routines developed by the National Renewable Energy Laboratory (NREL) were applied to evaluate the accuracy of results. This in-house code performed well for a NREL 5-MW baseline wind turbine [13]. It can be concluded that three approaches, including CFD, UBEM, and FAST, displayed good correlations in the unsteady aerodynamic analysis of a FOWT undergoing periodic pitching.

Figure 2. Platform pitching motion and its effects on the surrounding flow-field.

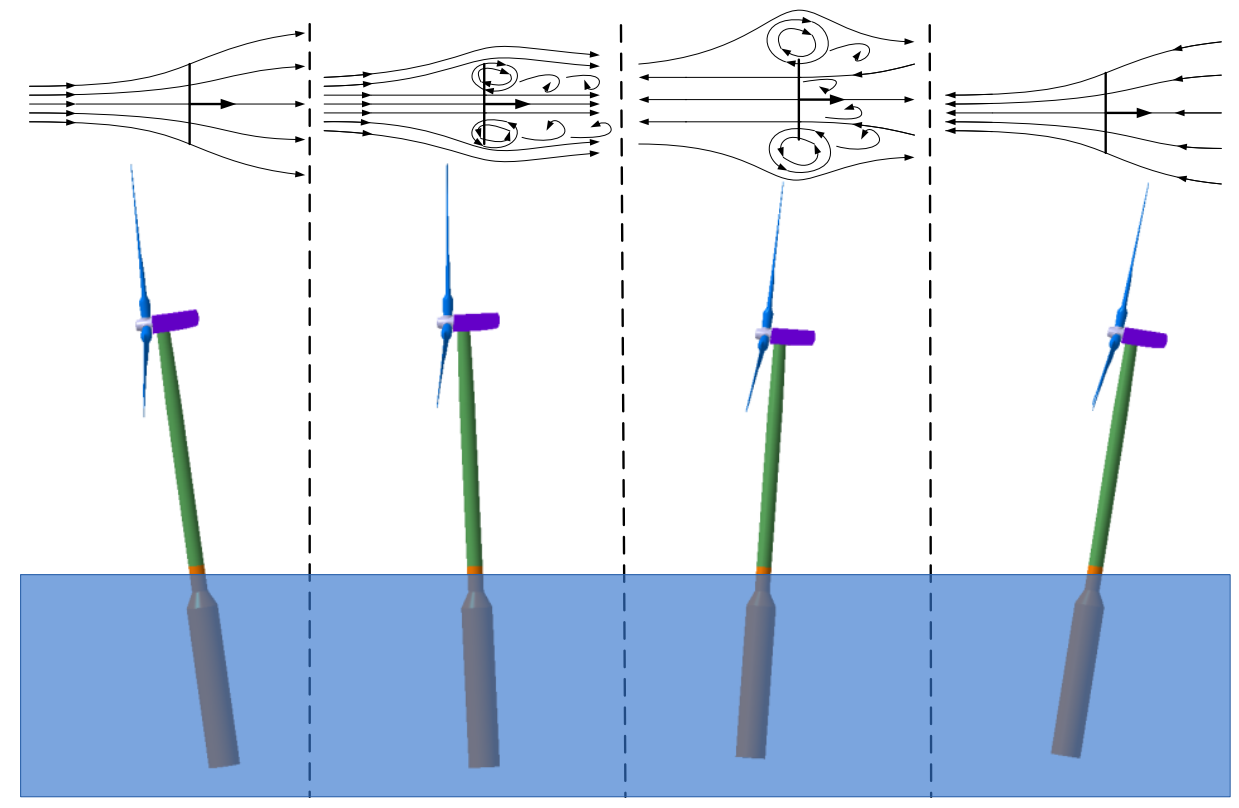

\section{Methodology}

The objective of the present study is to apply and analyse a computational fluid dynamic model. The three-dimensional, unsteady Reynolds-averaged Navier-Stokes equations with $k-\omega$ SST turbulence model were executed in the CD-Adapco STAR-CCM+ software package (Melville, NY, USA). This commercial code that is based on a finite volume approach allows researchers to perform fluid dynamic formulations, solutions algorithms, meshing schemes and post-processing. 


\subsection{Computational Fluid Dynamic Approach}

A segregated flow model solves the flow equations (one for each component of velocity, and one for pressure) in a segregated, or uncoupled, manner. All current simulations employed a semi-implicit method to solve the pressure-linked equations (SIMPLE) in which the linkage between the momentum and continuity equation is achieved through a predictor-corrector approach [14]. The second-order upwind scheme was applied for the convection term. This method has been shown to be an efficient means of solving the incompressible Reynolds-averaged Navier-Stokes (RANS) equations. In unsteady simulations, a second-order central difference scheme was used for temporal discretization. To obtain the aerodynamic power and thrust of unsteady simulations of the wind turbine blade and hub, a special original user field function has been developed. A similar approach to the user field function was adopted to extract the aerodynamic blade root load during the simulation of the pitching motion of a wind turbine. A simple user field function was also created to define the simple pitching motion of an offshore wind turbine platform. Rigid Body Motion (RBM) is applied to define the real motion of a wind turbine blade under each specific motion. The superposing motions technique was applied to define the sine function of the pitching motion of a platform.

\subsection{Overset Mesh Methods}

Overset meshes, which are sometimes also referred to as overlapping or chimera grids, are composed of a series of finite grid-volume meshes that overlap each other to form a single computational domain. The main advantages of this method are its ability to handle the complex geometries and the relative motion of bodies in dynamic simulations. Therefore, optimization studies can be performed. A detailed discussion of the overset mesh methodologies in STAR-CCM+ was presented by Hadzic [15]. In general, the computational domain is composed of a background grid and one or more small overset grid domains. The simulations can be employed by interpolating the active, inactive or acceptor cells depending on their position inside the computational domain. Whereas the discretized governing equations are simultaneously solved in active cells, no solution is computed for inactive cells. The fluxes through the cell face between the last active cell and the acceptor cell are approximated in an identical way as between two active cells. Acceptor cells are located between active and inactive cells of a given region. The tight coupling of the overset and background regions allows for a solution that is within an arbitrary low level of iteration errors. The rate of convergence of the iterative solution method is therefore similar to that of a single mesh of an identical resolution.

As we mentioned above, dynamic moving overset grids is one of the methods that allow for computation of bodies with relative motions, particularly the rotating motion of wind turbine blades. Several authors have performed CFD computations of wind turbines using the overset grids method. Li et al. [16] and Zahle et al. [17] computed the NREL Phase VI turbine at different wind speeds and yaw angles using the finite volume solver CFD Ship-Iowa and EllipSys3D, respectively. The 3-D aerodynamic effects for wind turbine blade plus tower and nacelle were investigated. These fully viscous, incompressible RANS flow solvers with overset grid showed a good agreement with experiment. For a large scale wind turbine as NREL 5-MW baseline wind turbine, OVERFLOW2 [18] and EllipSys3D [19] solver were applied to characterize the aerodynamic performance. Using the 
overset grids approach, Chow et al. [18] examined the aerodynamic characteristics of various passive geometric modifications at the inboard blade region of the wind turbine blade. It should be noted that most studies consider the rotation of the turbine blade of onshore or fixed-bottom wind turbines. The CFD simulation of floating offshore wind turbine blades is still challenge because of the coupled wind-wave condition that often leads to a highly unsteady flow condition. Under the coupling of wind and wave conditions [20], a FOWT should solve the interaction between fluid flow including the air-flow and sea water and wind turbine structures. Therefore, the fluid-structure interaction simulation for a full FOWT should be constructed in order to take the high unsteadiness into account. Generally, a similar strategy for this simulation can be referred from Hou et al. [21] and Viré et al. [22]. Applying the simulation of fluid-structure interaction, a full FOWT is expected to accurately and practically predict the aerodynamic loads under the harsh environmental conditions of an offshore area.

\section{Simulation Conditions}

\subsection{Computational Modeling of a Wind Turbine Blade}

The NREL 5-MW baseline wind turbine [23] model is a representative utility-scale wind turbine that is a suitable floating offshore wind turbine. The diameter of the rotor is $126 \mathrm{~m}$. The cross sections of the rotor blade are composed of series of DU (Delft University) and NACA (National Advisory Committee for Aeronautics) $64 \mathrm{xxx}$ airfoils from the hub to the tip of the out-board section. Related airfoil data can be found in Bazilevs et al. [24]. A full three-bladed rotor with overset Cartesian grid topology is explicitly modeled as shown in Figure 3. The hexahedral computational domain is applied with dimension of $1000 \mathrm{~m} \times 600 \mathrm{~m} \times 370 \mathrm{~m}(L \times W \times H)$, and extends 5- and 11-times the rotor radius in the negative (upstream) and positive (downstream) $\mathrm{x}$-direction, respectively.

Figure 3. (a) Illustration of the computational mesh domain; (b) Zoon-in refine overset grid area; (c) A view of layer grid adjustment blade surface; and (d) Blade surface tip area.

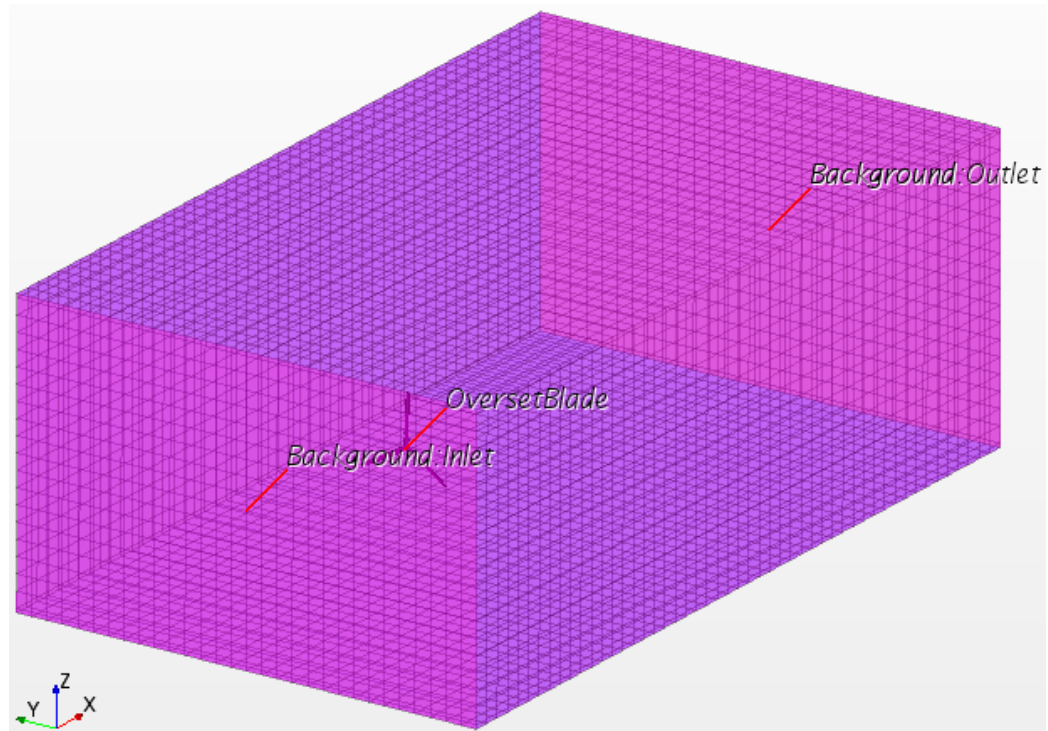

(a)

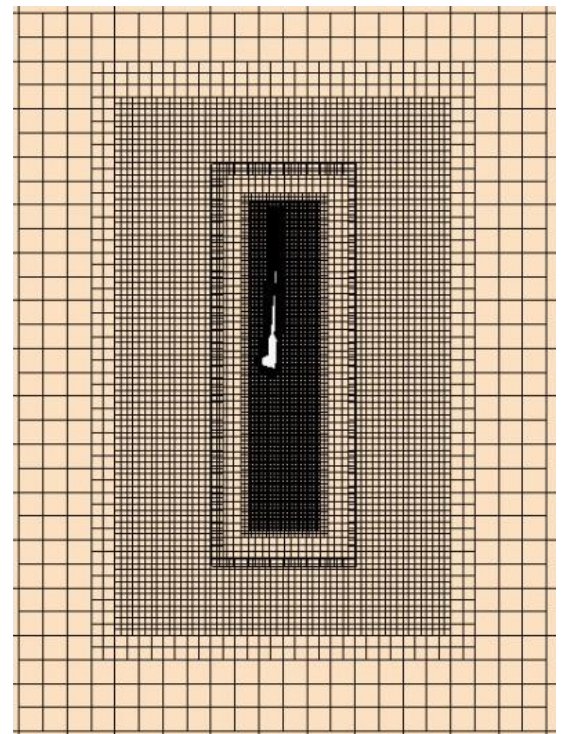

(b) 
Figure 3. Cont.

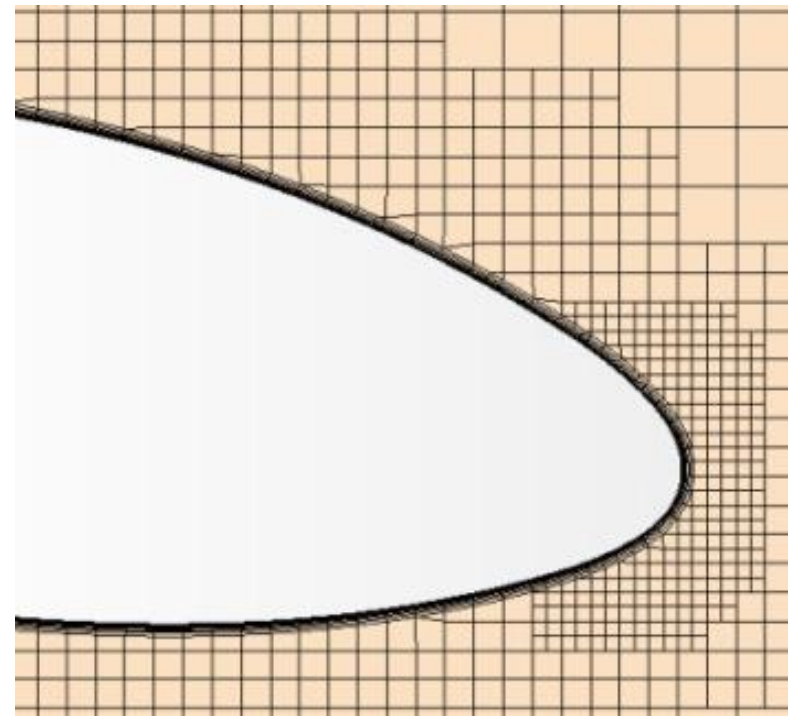

(c)

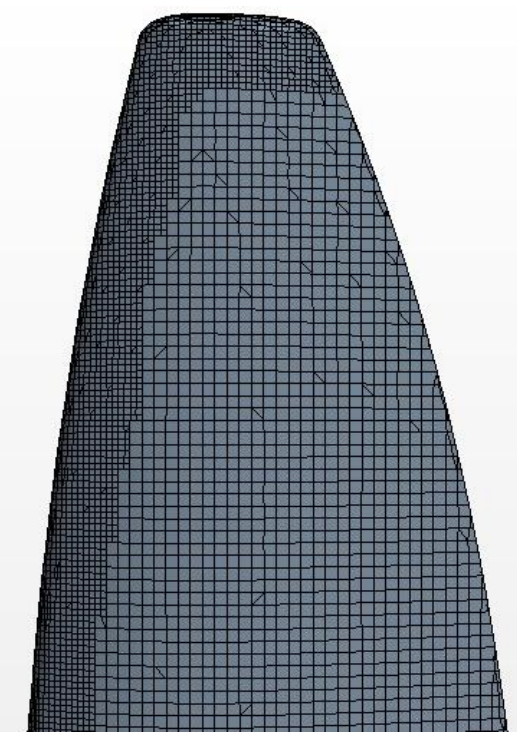

(d)

Refine mesh was generated behind the wind turbine rotor to capture wake behaviour. For blade surfaces, a refine grid is generated at the leading edge with a maximum and minimum grid size. The different sizes on the wind turbine surface led to a large variation of the amount of elements on the present computational domain as shown in Table 1. Near the rotor surface, we generated 10 layers of refined mesh; the total thickness of these layers was $2 \mathrm{~cm}$, and the progression factor was 1.2. This mesh structure for the rotor surface and adjacent mesh layer can be determined [24]. The total number of cell in the numerical grid was illustrated in Table 1, and the numerical grid consisted of the overset grid and background grid.

Table 1. The number of elements depending on the mesh size of wind turbine blade surface.

\begin{tabular}{ccccc}
\hline CFD Mesh Type & Case 1 & Case 2 & Case 3 & Case 4 \\
\hline Maximum size (m) & 0.030 & 0.024 & 0.015 & 0.012 \\
Maximum size (m) & 0.090 & 0.072 & 0.045 & 0.036 \\
Total number of elements (Million) & 6.8 & 9.7 & 16.9 & 23.7 \\
\hline
\end{tabular}

\subsection{Boundary Condition}

At the upstream boundary of the inlet, the velocity of a uniform axial flow was specified, and at the downstream boundary outlet, the static pressure was assumed to be at sea level pressures. On the surface of blades, a non-slip condition was defined. The symmetric boundary condition was imposed on the far field region of the computational domain. The Rigid Body Motion (RBM) technique was utilized to simulate moving rotor blade. To impose a pitching platform motion as a sine function in Equation (1) and Figure 4, superimposed motion is employed. The rotating center of the pitching motion was assumed to be $90 \mathrm{~m}$ below the hub center in the $z$-direction. However, in different real-platform designs, this center can be located at different positions. Additionally, the shear-stress transport (SST) $k$ - $\omega$ turbulence model was applied to account for turbulence flow effects. 
All computations considered a freestream velocity of $11 \mathrm{~m} / \mathrm{s}$, a constant rotating speed of $12 \mathrm{rpm}$ and, and a constant blade pitch angle of $0^{\circ}$ :

$$
\theta_{\text {pitch }}=\operatorname{Amp} \cdot \sin (2 \pi \cdot \text { Frep } \cdot \mathrm{t})
$$

The steady state computation was first performed for approximately 1000 iterations to have a convergence below $10^{-3}$, and then the unsteady computation used the steady state flow solution as the initial condition. The blade completed one cycle of revolution in $5 \mathrm{~s}$ with a time step size of $0.02778 \mathrm{~s}$ that corresponded to a blade rotation of $2.0^{\circ}$. All computations were effectively performed on a personal clustered of parallel machines that had 6-CPU Intel i7 processor, and 64 GB of RAM. For 10 sub-iterations of each global time step, the computation approximately required 3 min of $12 \mathrm{CPU}$ parallel processing time.

Figure 4. Platform pitching angle profile $(\mathrm{Amp}=4.0 \mathrm{deg}$, Freq $=0.1 \mathrm{~Hz})$.

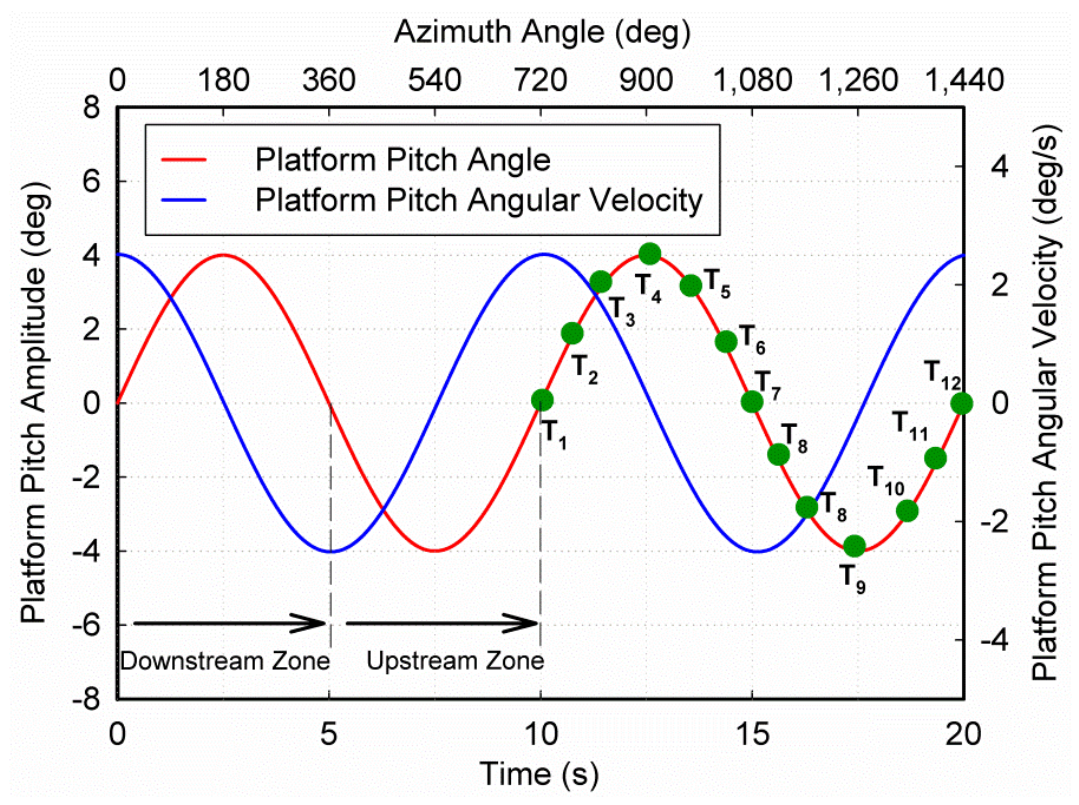

\section{Results and Discussion}

In this study, the unsteady simulation has performed using a computational fluid dynamic with multiple reference frames (CFD-MRF) model. This approach was conducted using the commercial ANSYS FLUENT version 14.0 software [13]. The Multiple Reference Frames (MRF) approach was applied to define the virtual blade rotation about its axis whereas the dynamic mesh and remeshing technique was used to prescribe the pitching motion of the floating platform. On the contrary, the computational fluid dynamic with rigid body motion (CFD-RBM) approach was applied to define the real blade rotation. Using the CFD-RBM approach, the flow unsteadiness due to wakes among wind turbine blades can be taken into account. To impose the pitching motion of the floating platform, a superimposing motion was also used in the commercial STAR-CCM+ software package. Figure 5 shows a computational road map using both the unsteady CFD-MRF and CFD-RBM approaches. The right hand side blocks of each road map indicate that the unsteady computational simulation was solved by the commercial solvers, whereas other computational blocks are developed by user-defined functions that were simultaneously incorporated in the commercial software. The main purpose of 
user-defined functions is to calculate the rotating center and rotating axis of wind turbine blades. Additionally, the axis vectors of the blade coordinate system were also calculated using the standard 3-2-1 Euler angle so that the force components on each blade root were estimated during the unsteady CFD simulation.

Figure 5. Computational flowchart for computational fluid dynamic (CFD) approaches.

(a) Computational fluid dynamic with multiple reference frames (CFD-MRF); and

(b) Computational fluid dynamic with rigid body motion (CFD-RBM).

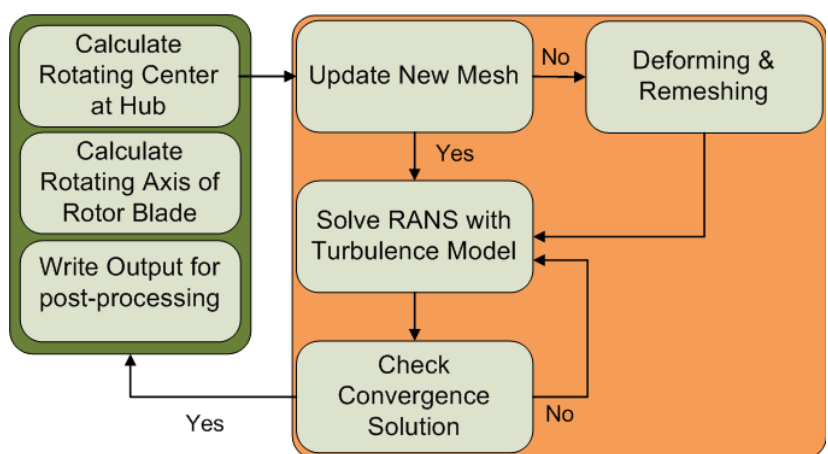

(a)

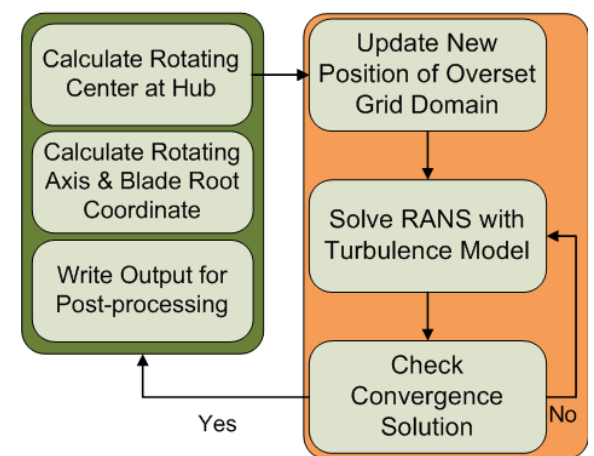

(b)

Generally, numerical solutions obtained by the CFD technique can be somewhat different depending on the discretization resolution of the computational domain. By limiting a maximum and minimum size of the elements on a wind turbine blade in Table 1, further refinement of the mesh over the computational domain was achieved in the present study. In order to show the numerical convergence of the present solution, the aerodynamic power and thrust over a range of an amount of elements are compared with those by the FAST code considering both blade element momentum (BEM) and generalized dynamic wake (GDW) models. As shown in Figure 6, the present CFD-RBM results show overall good correlations with those obtained by the FAST code.

Figure 6. Mesh convergence study for the grid size on NREL 5-MW wind turbine surface.

(a) Aerodynamic Power; and (b) Aerodynamic Thrust.

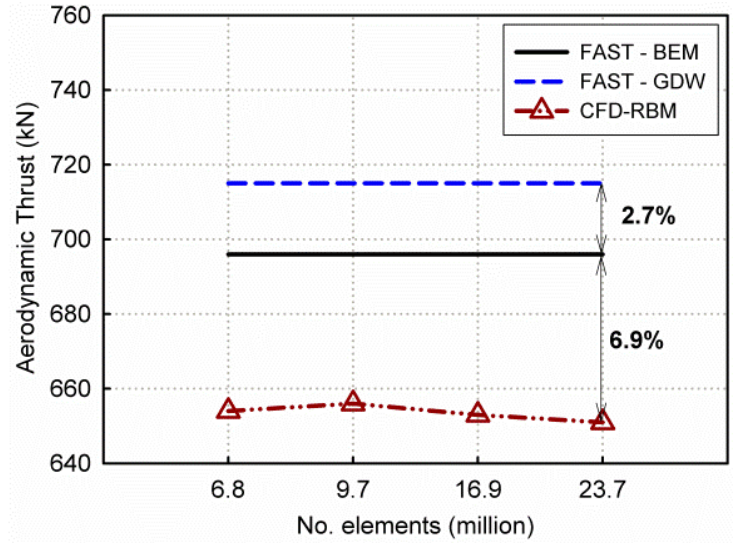

(a)

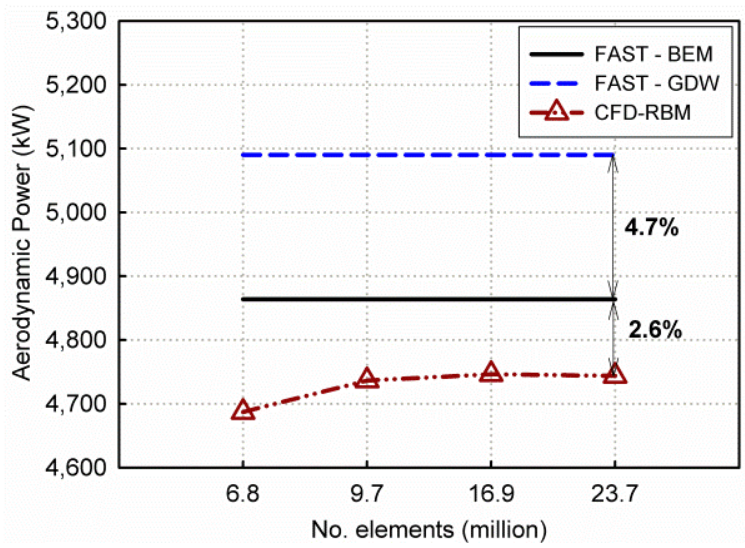

(b)

The present CFD approach tends to somewhat under predict since the mesh quality of the CFD method is very fine compared to the FAST code approach. It can be found that there are about $2.6 \%$ 
and $6.9 \%$ differences in the predicted aerodynamic thrust and power between the present CFD method and the FAST code, respectively, whereas, differences of aerodynamic power and thrust between FAST with BEM and GDW are about $4.7 \%$ and $2.7 \%$, respectively. Based on this mesh convergence test and fundamental comparisons we conducted more high level computations considering the pitching motion of a FOWT model. It should be emphasized here that flow unsteadiness for the condition of pitching motion for rotating blades must be higher than that of the previous comparison case. Thus, the computational grid of case 3 in Table 1 is chosen for the unsteady simulation of rotating wind turbine blades with platform pitching motion in order to accurately capture complex wake effect behind the rotating blades. The refinement grid on a part of the trailing edge of wind turbine blade can be seen in Figure 3d. Figures 7 and 8 show the comparison of the unsteady aerodynamic power and thrust responses among the CFD, UBEM and FAST solvers for the pitching motion with a frequency (Freq) of $0.1 \mathrm{~Hz}$ and the amplitude (Amp) of $1^{\circ}$ and $4^{\circ}$. These frequencies and amplitudes for the pitching motion may exist under normal wind and wave conditions experienced by the floating wind turbine. The aerodynamic power and thrust respond as a sine function. Figures 7 and 8 show that the predicted aerodynamic responses are similar to the frequency of the pitching motion of a FOWT which periodically moves with a frequency of $0.1 \mathrm{~Hz}$. For the initial platform pitching motion, a numerical error exists because of quickly changing wind conditions. After this initial time, the dynamic responses regularize in all of the considered numerical approaches. During the $1 / 4 T$ and $3 / 4 T$ period of the pitching motion, the angular velocity decreases as shown in Figure 4. Therefore, this lower angular velocity decreases the contribution of additional velocity on a given rotor blade and increases the non-axial angle of the wind turbine. By contrast, during the 2/4T and $4 / 4 T$ time of the pitching motion, additional velocity contributions tend to increase, whereas the non-axial (yaw/pitch) angle error decreases. The decrease and increase of the additional velocity contribution and non-axial angle are present in both the downstream and upstream regimes of the rotor blade. As shown in Figures 7 and 8, increasing the additional velocity contribution and decreasing the non-axial angle increase the aerodynamic power and thrust for the $1 / 4 T$ time of pitching motion. However, this response reverses during the 3/4T time. The wind turbine blades substantially move in the wake regime (downstream) during the $1 / 4 T$ time whereas there exists a wake zone during the 3/4T time. Alteration in the additional velocity contribution and the strong interaction between the rotor blade and wake regime significantly affect the aerodynamic performance of a wind turbine. During the $1 / 4 T$ time, the aerodynamic power and thrust tend to increase because of increases on the additional velocity contribution and the strong interaction between the rotor blades and wake regime. However, a similar behavior is not exhibited during the 4/4T time while additional velocity contribution decreases and strong interaction between rotor blades and wake regime remains. Therefore, increasing the additional velocity contribution (and not wake interference) significantly affects the performance during the 1/4T time, and the reverse is true during the 4/4T time. For weak wake interactions between the rotor blades and its wake during the $2 / 4 T$ and $3 / 4 T$ time, the additional velocity contribution and non-axial angle maintain a key role in the aerodynamic performance. The increasing or decreasing in additional velocity contribution increases or decreases the aerodynamic thrust and power, respectively.

As shown in Figures 7 and 8, the current numerical approaches predicted a good trend for the aerodynamic power and thrust of a FOWT under pitching conditions. The UBEM approach over-predicted these values more than the other approaches. The FAST with BEM tends to 
underestimate the aerodynamic power and thrust compared to the FAST with GDW, especially the peak area of additional velocity contribution as shown in Figure 9. The results obtained by the CFD-RBM approach displays a difference in the maximum peak area in which the pitching motion achieves a maximum motion velocity. Additionally, the maximum power occurs at a $4^{\circ}$ motion amplitude. At that amplitude, the relative wind velocity contribution also achieves a maximum magnitude. The UBEM code did not account for the effects of wake interference between the rotor blade and windmill in present study, whereas FAST solver can account for wake dynamics, particularly the GDW aerodynamic theory. Both the axial and tangential induction factor will be simultaneously applied by the AeroDyn of FAST solver.

Figure 7. Unsteady aerodynamic comparison using the UBEM, CFD and FAST (NREL) methods for a platform pitching motion with an amplitude of $1^{\circ}$ and a frequency of $0.1 \mathrm{~Hz}$.

(a) Aerodynamic Power; and (b) Aerodynamic Thrust.

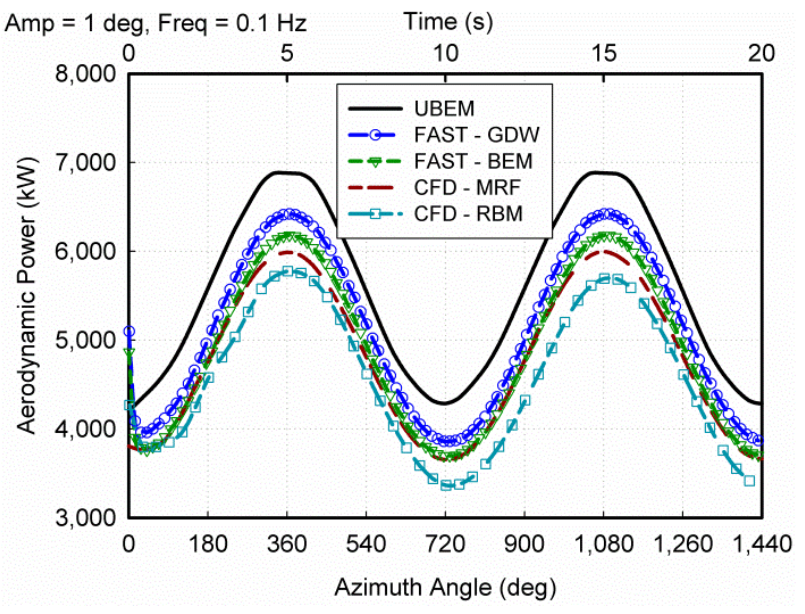

(a)

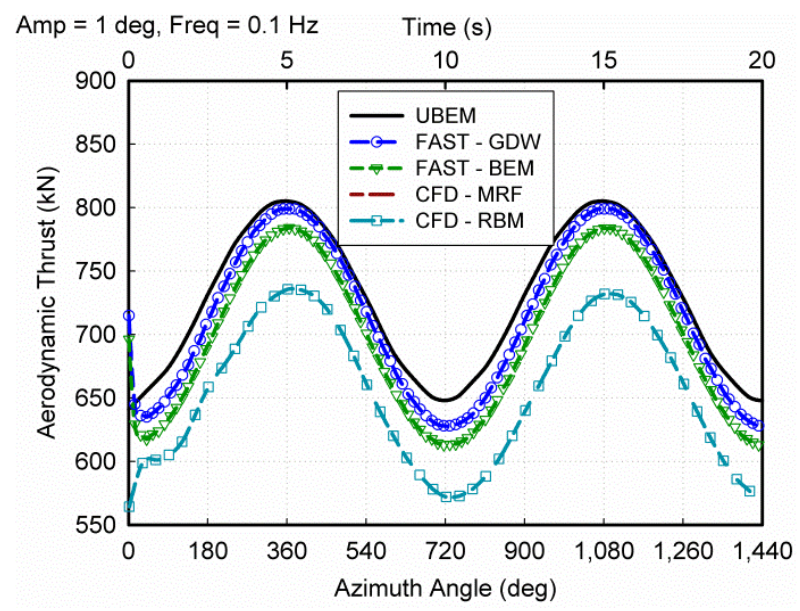

(b)

Figure 8. Unsteady aerodynamic comparison using the UBEM, CFD and FAST (NREL) methods for a platform pitching motion with an amplitude of $4^{\circ}$ and a frequency of $0.1 \mathrm{~Hz}$. (a) Aerodynamic Power; (b) Aerodynamic Thrust.

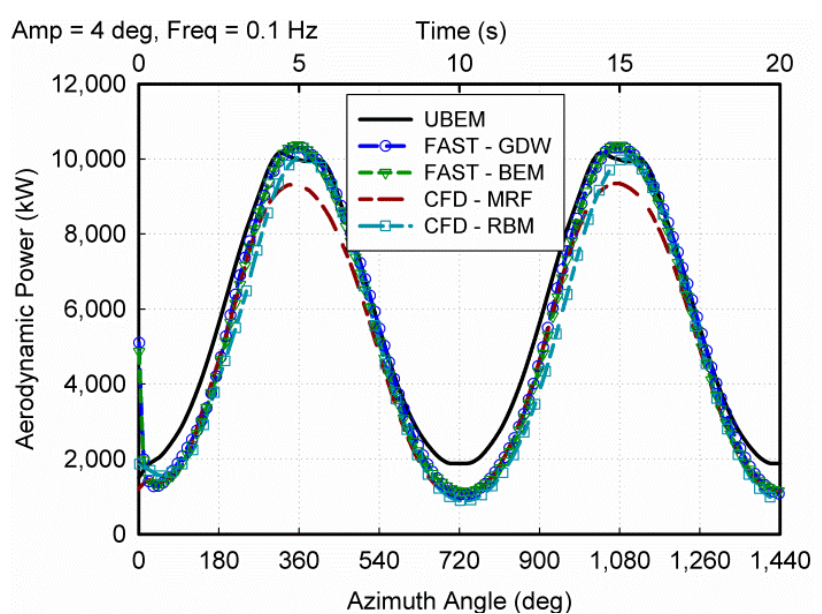

(a)

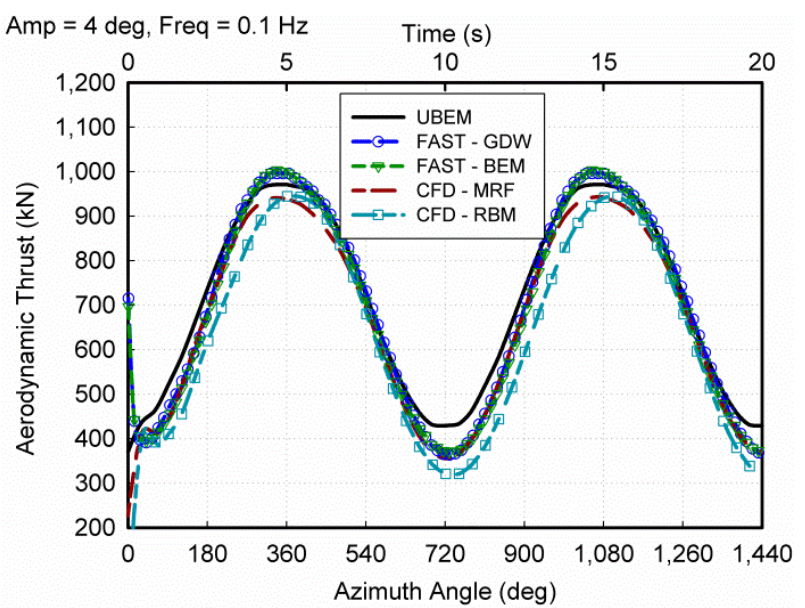

(b) 
Figure 9. Aerodynamic comparisons using the UBEM, CFD and FAST (NREL) methods for different pitching amplitudes of a FOWT (Freq $=0.1 \mathrm{~Hz})$.

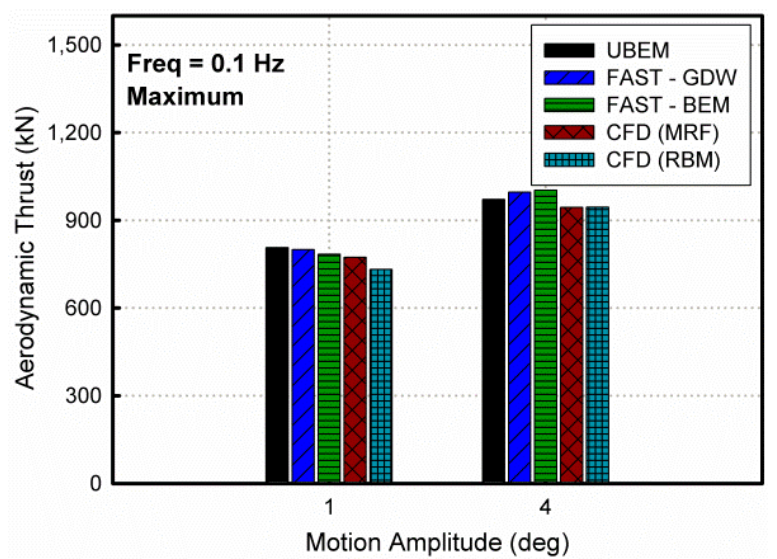

(a)

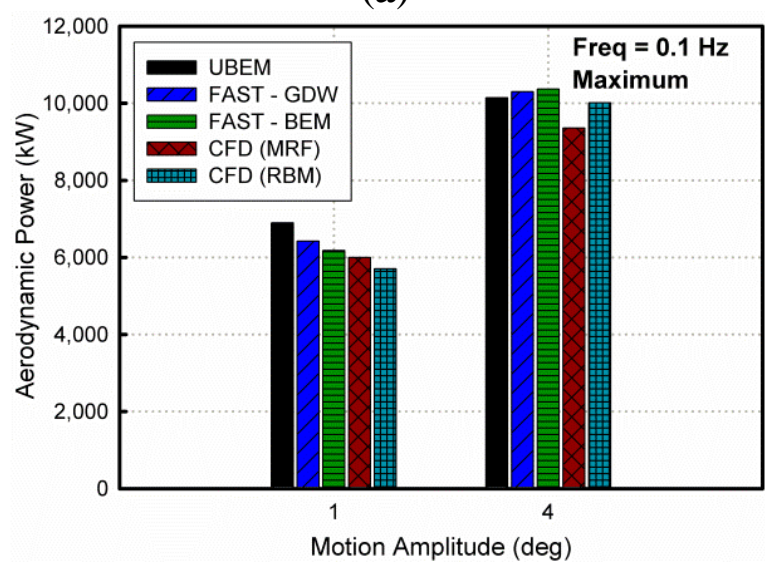

(c)

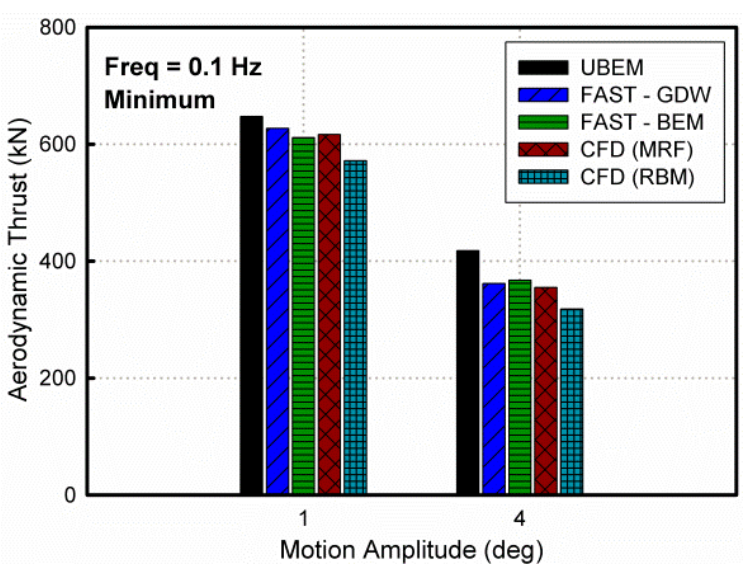

(b)

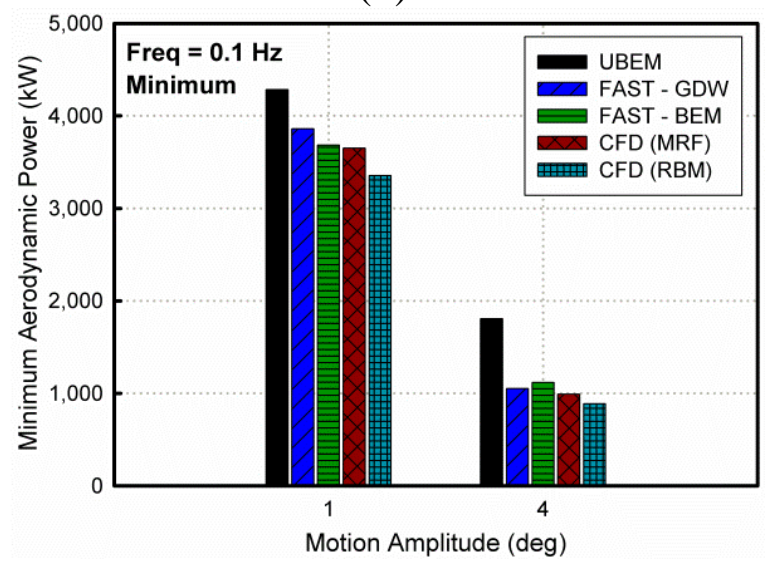

(d)

The GDW aerodynamic theory, which is based on a potential flow solution to Laplace's equation, can be modeled with more flow states and a fully nonlinear implementation to account for the turbulence in and spatial variation of the inflow [25]. However, the unsteady CFD can effectively predict the aerodynamic wake effect better than other numerical approaches. This method completely considers the viscous effect and vortex wake at the interference regime between the rotor blade and windmill. Particularly, an unsteady CFD-RBM approach that models real blade rotation about a hub center can accurately account for the flow field interaction between the blades and wake regime. Therefore, the unsteady CFD-RBM approach is expected to produce the lowest estimation of aerodynamic power and thrust responses because these values are over-predicted by the UBEM solver and other models.

As shown in Figure 9, the aerodynamic power and thrust will increase with increasing amplitude of the pitching motion. At the maximum peak of aerodynamic power curves, the unsteady CFD-RBM predicted 5.7 MW and 10.0 MW of power for the different motion amplitudes of $1^{\circ}$ and $4^{\circ}$, respectively. Compared to normal operating conditions for the wind turbine without wave effects, the aerodynamic power increased by approximately $14 \%$ and $100 \%$ for these respective amplitudes. This is a significant increase for a large wind turbine system. However, the aerodynamic thrusts obtained by unsteady CFD-RBM at maximum peak zone tend to slightly differ for $1^{\circ}$ of amplitude pitching motion. The thrust increases to approximately $30 \%$ for a $4^{\circ}$ amplitude pitching motion. In the 
UBEM simulation, a large variation in the aerodynamic load responses also appears for the different amplitudes. The aerodynamic power and thrust responses are therefore dominated by the frequency motion of the FOWT.

Figure 4 shows a typical pitching amplitude of the platform motion with reference to time or the azimuth angle of the rotor blade. The turbine rotor instantaneous position because of this pitching motion is illustrated in Figure 10.

Figure 10. Visualisation of instantaneous computed vortices for unsteady pitching of a FOWT simulation represented by an isosurface of vorticity $\left(\operatorname{Amp}=4^{\circ}\right.$, Freq $\left.=0.1 \mathrm{~Hz}\right)$.

(a) Downstream zone (Top: $\mathrm{T}_{1} \rightarrow \mathrm{T}_{6}$ ) and (b) Upstream zone (Bottom: $\mathrm{T}_{7} \rightarrow \mathrm{T}_{12}$ ).

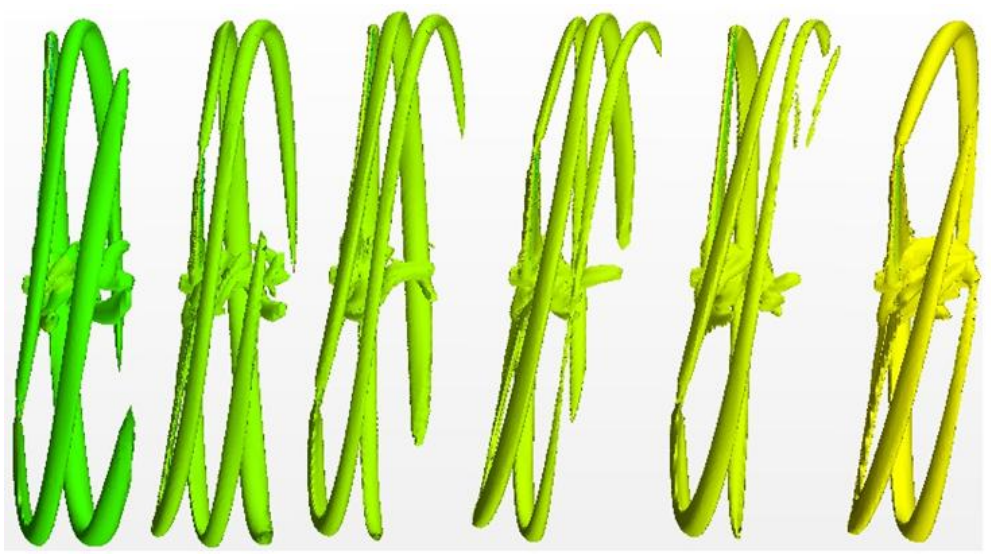

(a)

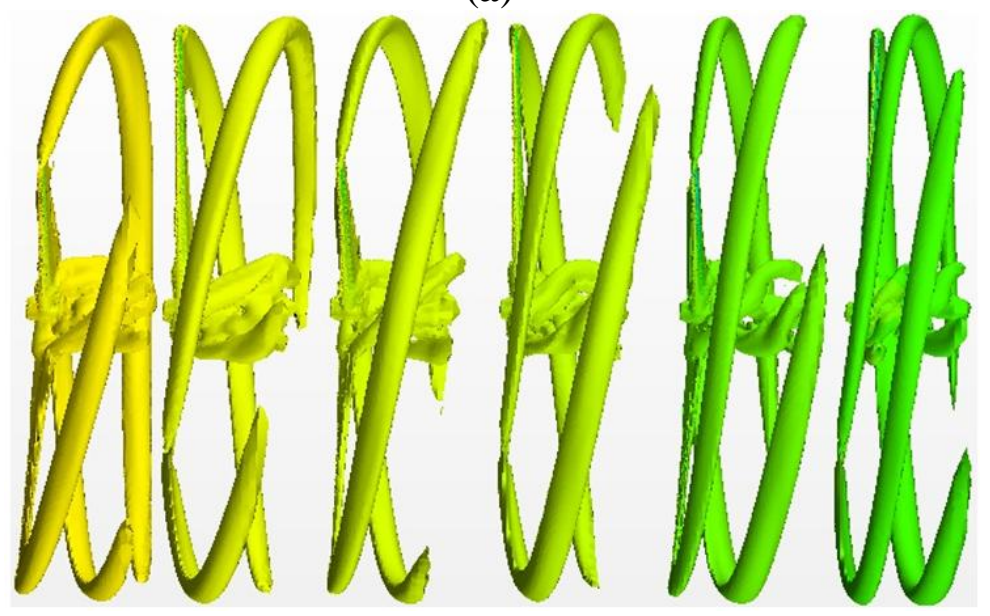

(b)

The computed iso-surfaces show the presence of instantaneous vortices beyond the wind turbine rotor during prescribed pitching motion. A side-view of the vortices is visualized. The blade tip vortices are strong and stable. Strong vortices also detach from the roots of the blades at which the geometry changes quickly from a DU40 airfoil profile to cylindrical posts attached to the hub. The vortical structures dissipate rapidly away from the regions covered by the grid refinements, (downstream of the rotor plane below 1 blade length as shown in Figure 3). This complex physical flow can only be captured in the advanced CFD method. In the downstream region, the rotor blades deeply move in the wake zone, and they subsequently break off. The gaps among tip vortices showed that a strong interaction among the rotor blades and wake regimes appeared in the current study. The 
break-off of wake from the rotor blade and the increasing additional velocity contribution minimized vortices behind the rotor blade. The magnitude of wake also decreases as shown in the vortices view of the downstream zone. Similar behaviors in the wake pattern exist when rotor blades move in upstream regions. The gaps among tip vortices continue to increase because plane rotation still moves forward. Subsequently, the gaps decrease because rotor blades move downward. In this case, the wake magnitude increases as shown in the vortices view of the upstream zone. The unsteady CFD simulation tool is one of the best methods that can most accurately analyze the complex flow around the FOWT. The physical flow patterns can also be captured and visualized using the unsteady CFD method for physical investigation. It seems from the results that the conventional blade element momentum (BEM) theory needs to be additionally improved to account for the complex effect of induced velocity due to the free motion of the floating wind turbine. Typical integrated-aerodynamic loads on each wind turbine blade at the blade root are shown in Figure 11. Blade \#1 is located at 0 o'clock position on a clock since the wind turbine rotor initiates in pitching motion. To calculate the blade load based on the blade coordinate system, a special user field function has been originally developed based on the multiple reference frames algorithm. As shown in Figure 11, all aerodynamic blade loads on each of the wind turbine blades varies as a sine function with a similar frequency of pitching motion. Because of blade position has the longest distance between the pitching rotating center and the local span position of the rotor blade, blade \#1 is predicted to have the highest aerodynamic load due to highest additional velocity contributions, which is directly proportional to the angular velocity and distance.

Figure 11. Unsteady aerodynamic load on the blade coordinate system by the CFD-RBM approach $\left(\right.$ Amp $=4^{\circ}$, Freq $\left.=0.1 \mathrm{~Hz}\right)$.
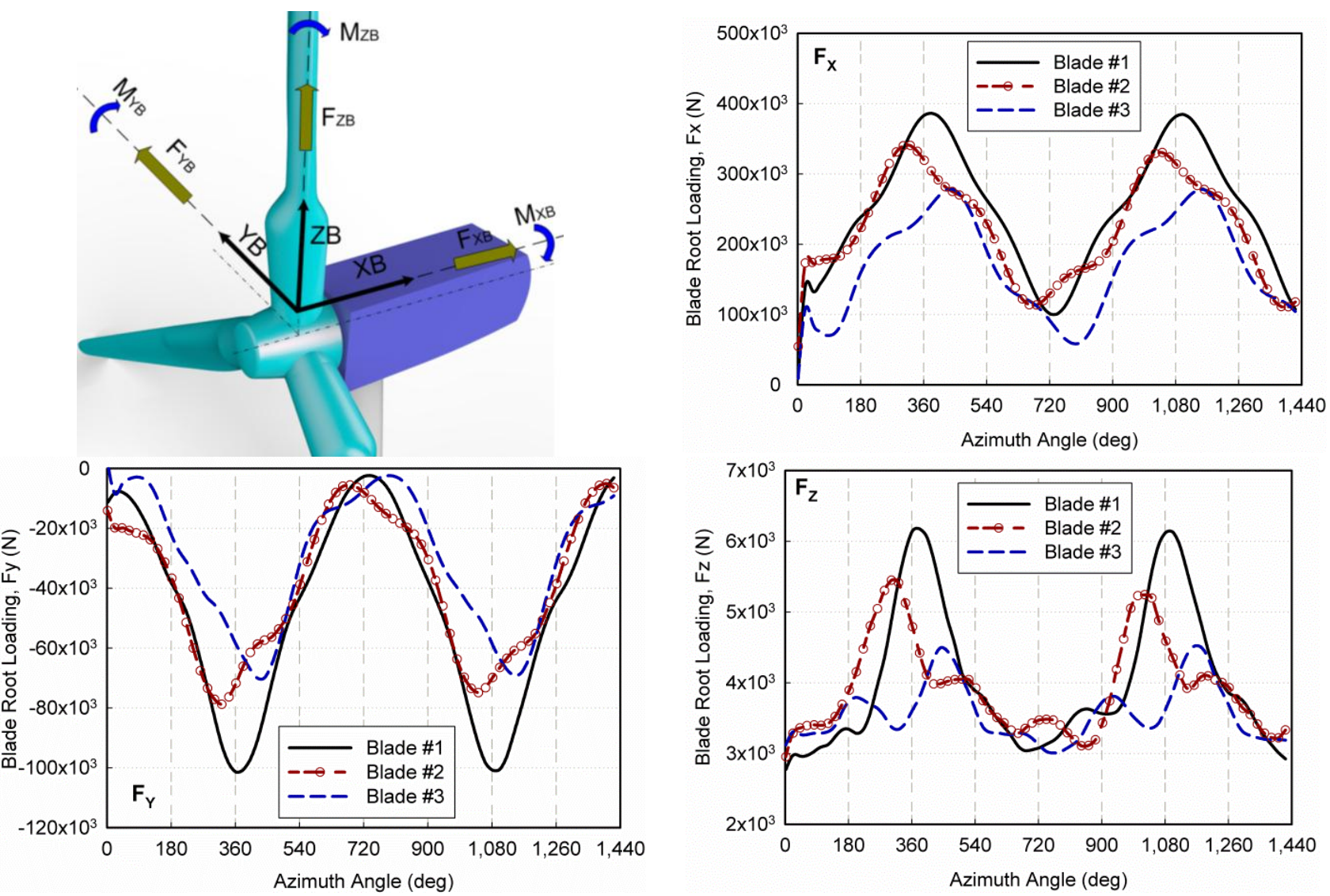


\section{Conclusions}

The unsteady flow field analysis around the rotating blades of a floating offshore wind turbine is a complex process that is simultaneously influenced by aerodynamics, ocean waves, sea current, hydrodynamics, and other factors because the support platform of the wind turbine system has six degrees of freedom motion. Aerodynamic processes greatly affect the floating wind turbine and to a lesser extent, the floating motions of the support platform, because the tower is tall. In this study, a complex unsteady aerodynamic analysis on the rotating rotor blades of a floating support platform with the motion of the supporting offshore platform has been successfully conducted using computational fluid dynamics including rigid body motion and overset grid techniques. Aerodynamic loads on the blade are greatly altered because of its floating motion. This flotation can also lead to substantial variation in the generated power by the wind turbine. Although, the present study primarily showed the periodic pitch motion of the support platform of the floating wind turbine system, the present method can be applied to conduct accurate analysis of a generic horizontal and vertical-axis FOWT models. It is practically shown that the proposed equivalent averaged velocity method (EqAM) agreed well with the direct local relative velocity method (DLRM). Three numerical approaches, including UBEM, FAST with BEM and GDW, have been used for the numerical comparisons. All numerical tools show good agreement over the results of the aerodynamic power and thrust for small pitching motion amplitudes. The differences in the predictions of UBEM, FAST with BEM and GDW are moderately increased for an increased oscillating amplitude of $4^{\circ}$. These traditional numerical approaches have several limitations because their assumptions over-predict when compared to the unsteady CFD approaches. Thus the present numerical method has a strong potential to more accurately predict unsteady aerodynamic loads for the typical offshore wind turbine blades, even with arbitrary motions of a floating support platform.

\section{Acknowledgments}

This work was partially supported by the Human Resources Development Program (No. 20114010203070) of the Korea Institute of Energy Technology Evaluation and Planning (KETEP) Grant funded by the Korea government Ministry of Trade, Industry and Energy, and also by Korea Institute of Machinery and Materials (KIMM).

\section{Conflicts of Interest}

The authors declare no conflict of interest.

\section{References}

1. Sebastian, T.; Lackner, M.A. Analysis of the induction and wake evolution of an offshore floating wind turbine. Energies 2012, 5, 968-1000.

2. Sebastian, T.; Lackner, M.A. Development of a free vortex wake method code for offshore floating wind turbines. Renew. Energy 2012, 46, 269-275.

3. Sebastian, T.; Lackner, M.A. Characterization of the unsteady aerodynamics of offshore floating wind turbines. Wind Energy 2012, 16, 339-352. 
4. Sebastian, T. The Aerodynamic and Near Wake of an Offshore Floating Horizontal Axis Wind Turbine. Ph.D. Thesis, University of Massachusetts, Amherst, MA, USA, February 2012.

5. Peters, D.; Chen, S. Momentum theory, dynamic inflow, and the vortex-ring state. J. Am. Helicopter Soc. 1982, 27, 18-24.

6. Jeon, M.; Lee, S.M.; Lee, S.G. Unsteady aerodynamics of offshore floating wind turbines in platform pitching motion using vortex lattice method. Renew. Energy 2014, 65, 207-212.

7. Matha, D.; Wendt, F.; Werner, M.; Cheng, P.W.; Lutz, T. Aerodynamic inflow conditions on floating offshore wind turbine blades for airfoil design purposes. In Proceedings of the Twenty-second International Offshore and Polar Engineering Conference, Rhodes, Greece, 17-22 June 2012.

8. Jonkman, J.M. Definition of the Floating System for Phase IV of OC3; Technical Report NREL/TP-500-47535; National Renewable Energy Laboratory: Golden, CO, USA, 2010.

9. Sarun, B. Computational Studies of Horizontal Axis Wind Turbines in High Wind Speed Condition Using Advanced Turbulence Models. Ph.D. Thesis, Georgia Institute of Technology, Atlanta, GA, USA, December 2006.

10. Villalpando, F.; Reggio, M.; Ilinca, A. Assessment of turbulence models for flow simulation around a wind turbine airfoil. Model. Simul. Eng. 2011, doi:10.1155/2011/714146.

11. Carcangiu, C.E. CFD-RANS Study of Horizontal Axis Wind Turbines. Ph.D. Thesis, Università degli Studi di Cagliari, Cagliari, Italy, January 2008.

12. Tran, T.T.; Kim, D.H.; Bae, K.S. Extreme load estimation for a large wind turbine using CFD and unsteady BEM. In Computational Science and Its Applications-ICCSA 2013; Springer: Berlin, Germany, 2013; pp. 127-142.

13. Tran, T.T.; Kim, D.H.; Lee, J.H.; Song, J.S.; Lee, G.H.; Rim, C.W. Comparison of unsteady aerodynamic analysis methods for the blade with pitching motion of large floating offshore wind turbine. In Proceedings of the Fall Korea Wind Energy Association Conference, Busan, Korea, 25-27 November 2013.

14. CD-Adapco STAR-CCM+ v.8.06 Help Manual. Available online: http://www.cd-adapco.com/ products/star-ccm\%C2\%AE (accessed on 25 June 2014).

15. Hadzic, H. Development and Application of Finite Volume Method for the Computation of Flows Around Moving Bodies on Unstructured, Overlapping Grids. Ph.D. Thesis, Technical University Hamburg-Harburg, Hamburg, Germany, December 2005.

16. Chow, R.; van Dam, C.P. Inboard stall and separation mitigation techniques on wind turbine rotors. In Proceedings of 49th AIAA Aerospace Sciences Meeting, Orlando, FL, USA, 4-7 January 2011.

17. Li, Y.; Paika, K.J.; Xing, T.; Carrica, P.M. Dynamic overset CFD simulations of wind turbine aerodynamics. Renew. Energy 2012, 37, 285-298.

18. Zahle, F.; Sørensen, N.N.; Johansen, J. Wind turbine rotor-tower interaction using an incompressible overset grid method. Wind Energy 2009, 12, 594-619.

19. UPWIND, Aerodynamics and Aero-Elasticity Rotor Aerodynamics in Atmospheric Shear Flow. Available online: http://vbn.aau.dk/files/11558577/UpWind (accessed on 25 July 2013).

20. Viré, A. How to float a wind turbine. Rev. Environ. Sci. Biotechnol. 2012, 11, 223-226.

21. Hou, G.; Wang, J.; Layton, A. Numerical methods for fluid-structure interaction-A review. Commun. Comput. Phys. 2012, 12, 337-377. 
22. Viré, A.; Xiang, J.; Milthaler, F.; Farrell, P.E.; Piggott, M.D.; Latham, J.P.; Pavlidis, D.; Pain, C.C. Modelling of fluid-solid interactions using an adaptive mesh fluid model coupled with a combined finite-discrete element model. Ocean Dyn. 2012, 62, 1487-1501.

23. Jonkman, J.; Butterfield, S.; Musial, W.; Scott, G. Definition of a 5-MW Reference Wind Turbine for Offshore System Development; Technical Report NREL/TP-500-38060; National Renewable Energy Laboratory: Golden, CO, USA, 2009.

24. Bazilevs, Y.; Hsu, M.C.; Akkerman, I.; Wright, S.; Kakizawa, K.; Henicke, B.; Spielman, T.; Tezduyar, T.E. 3D simulation of wind turbine rotors at full scale. Part I: Geometry modeling and aerodynamics. Int. J. Numer. Methods Fluids 2011, 65, 207-235.

25. Moriarty, P.J.; Hansen, C.A. AeroDyn Theory Manual; Technical Report NREL/EL-500-36881; National Renewable Energy Laboratory: Golden, CO, USA, 2005.

(C) 2014 by the authors; licensee MDPI, Basel, Switzerland. This article is an open access article distributed under the terms and conditions of the Creative Commons Attribution license (http://creativecommons.org/licenses/by/3.0/). 